FORMATION Formation emploi

Revue française de sciences sociales

114 | Avril-juin 2011

Pêle-mêle

\title{
Des vertus et limites de la comparaison statistique internationale
}

José Rose

\section{(2) OpenEdition}

Journals

Édition électronique

URL : http://journals.openedition.org/formationemploi/3382

DOI : 10.4000/formationemploi.3382

ISSN : 2107-0946

Éditeur

La Documentation française

Édition imprimée

Date de publication : 31 juillet 2011

Pagination : 83-87

ISSN : 0759-6340

\section{Référence électronique}

José Rose, «Des vertus et limites de la comparaison statistique internationale », Formation emploi [En ligne], 114 | Avril-juin 2011, mis en ligne le 08 septembre 2011, consulté le 30 octobre 2020. URL http://journals.openedition.org/formationemploi/3382 ; DOI : https://doi.org/10.4000/

formationemploi.3382

(c) Tous droits réservés 


\section{Note de lecture}

\section{Des vertus et limites de la comparaison statistique internationale. Présentation de l'ouvrage de F. Dubet, M. Duru-Bellat, A. Vérétout, "Les sociétés et leur école: emprise du diplôme et cohésion sociale»}

par José Rose*

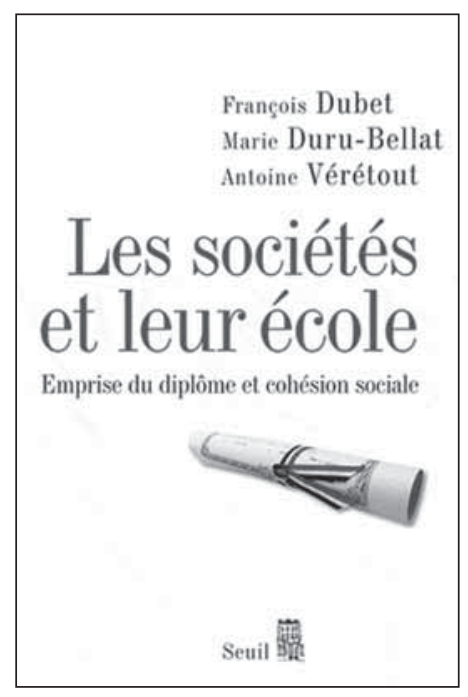

«La sociologie comparée n'est pas une branche particulière de la sociologie, c'est la sociologie même en tant qu'elle cesse d'être purement descriptive et aspire à rendre compte des faits »: cette citation, souvent tronquée sur la fin, d'E. Durkheim dans Les règles de la méthode sociologique (1895), évoque le passage de la description à l'interprétation. C'est l'une des questions posées par l'ouvrage de F. Dubet, M. Duru-Bellat et A. Vérétout, intitulé « Les sociétés et leur école, emprise du diplôme et cohésion sociale ».

«La comparabilité ne s'applique pas dans ce cas directement à des phénomènes (ou à des objets) particuliers comparés terme à terme, elle s'applique plutôt à des ensembles de phénomènes qui constituent dans leurs interdépendances des "cohérences" nationales propres à chaque pays » : ce point de vue de M. Maurice, F. Sellier et J.-J. Sylvestre, formulé dans Politique d'éducation et organisation industrielle en

* José Rose est professeur de sociologie à l'université de Provence, membre du LEST (Laboratoire d'économie et de sociologie du travaill-CNRS. II a notamment publié l'ouvrage collectif Les relations formation-emploi en 55 questions, paru chez Dunod en 2008 sous la direction de J.J. Paul et J. Rose ; "Penser les catégories et leurs frontières. Travail et études : équivalence, alternance et dissonances » paru dans un ouvrage collectif intitulé Les catégories sociales et leurs frontières, paru en 2011 aux Presses Universitaires de Laval sous la direction de A. Degenne, C. marry et S. Moulin.

Thèmes de travaux: relations formation-emploi, transitions professionnelles, transformations du travail, enseignement supérieur. 
France et en Allemagne. Essai d'analyse sociétale (PUF, 1982), concerne les comparaisons internationales. C'est l'un des problèmes soulevés par cet ouvrage comparant les sociétés et leur école.

Après avoir souligné l'actualité du sujet et l'intérêt de l'objet de recherche, on présentera les principaux résultats de cette recherche, puis on mettra en évidence quelques questions théoriques et méthodologiques avant de conclure sur le problème de l'interprétation de ces constats.

1. L'objet du livre, destiné aussi à un lectorat de non spécialistes, est d'importance et d'actualité puisqu'il s'agit de réfléchir à la cohésion sociale et à la reproduction des sociétés, à l'articulation entre école et société, entre la formation et les valeurs de confiance et de tolérance, entre cohésions scolaire et sociale. Il soulève ainsi des questions à la fois sociologiques et politiques.

Cette dimension politique du propos est clairement revendiquée par les auteurs lorsqu'ils suggèrent de «penser plus rationnellement les politiques scolaires » (p. 11), de « refuser le fatalisme et le volontarisme » (p. 104), lorsqu'ils observent « les illusions et les impasses du modèle de l'égalité des chances méritocratiques » (p. 189) et l'importance des styles éducatifs (communauté démocratique, communauté bienveillante, école du savoir, excellence pour tous) ou encore lorsqu'ils soulignent les limites de la scolarisation et la singularité de la France ("l'école n'y est efficace que pour une partie des élèves », p. 77). De fait, l'ouvrage peut stimuler la réflexion de ceux qui s'interrogent sur les réformes du système éducatif, le développement scolaire et ses limites ou la perpétuation des inégalités. Mais il peut aussi, et les auteurs le soulignent eux-mêmes, être source de malentendus dans une période où palmarès et bonnes pratiques (benchmarking) font florès.

L'ouvrage se propose d'analyser «la nature et la force des relations entre les systèmes scolaires et les sociétés » (p. 11). Il se construit à partir d'une comparaison des sociétés et de leur école dans vingt sept pays caractérisés, essentiellement à partir des données de l'OCDE (Organisation de coopération et de développement économiques), par «leur intégration (leur structure et leur degré d'égalité) et leur cohésion (les valeurs qui font lien) ». Et il se décline en six chapitres permettant de traiter successivement les points suivants : définition des termes d'intégration et de cohésion sociale (1) ; caractérisation des sociétés (2) et des systèmes éducatifs (3); liens entre les sociétés et leur école (4); emprise de l'école et reproduction sociale (5); emprise scolaire, cohésion et justice sociales (6).

Le point de vue explicatif central se développe autour de la notion d'emprise scolaire, celle-ci exprimant « le rôle joué par les hiérarchies scolaires dans la formation des hiérarchies sociales » (p. 131) ou encore «l'emprise du diplôme sur les positions sociales » (p. 133); elle se mesure par une variable agrégeant deux indices de l'avantage comparatif apporté par la formation sur le taux d'emploi et le niveau de salaire. Ceci conduit à mettre en évidence le rôle déterminant du marché du travail et des effets de la conjoncture, mais aussi du diplôme, dans la compréhension de la diversité des situations nationales.

Les sources et méthodes sont présentées de façon précise dans une annexe fort utile pour qui veut réfléchir à la méthodologie des comparaisons internationales. Les données proviennent pour la plupart de l'OCDE, notamment des enquêtes PISA (Programme for International Student Assessement) mais aussi d'autres enquêtes internationales (World Values Survey et International Social Survey Programme). La construction des indicateurs est également explicitée, ce qui permet de les discuter, et la tentative de construction de «macro-variables » permettant de caractériser de façon simple le niveau d'intégration et la cohésion sociales des sociétés ne manque pas de bravoure. Quant aux critiques susceptibles d'affecter ces données (absence d'homogénéité temporelle, diversité des instances de production des statistiques), elles ne sont pas passées sous silence, ce qui permet de raviver chez le lecteur les questions classiques de toute approche comparative - définition des concepts, construction des indicateurs, choix des nomenclatures, insuffisance des valeurs moyennes, etc. - et de l'inciter à une grande prudence dans l'interprétation des résultats. Se lancer dans un tel travail de comparaison de statistiques sur un aussi grand nombre de pays relève de la gageure mais il faut bien faire avec les outils dont on dispose, sauf à ne jamais pouvoir réaliser d'études comparatives à grande échelle. 
La démarche sociologique retenue est également fort utile. Il s'agit en effet d'une approche statistique, partagée par les sociologues pratiquant une macrosociologie quantitative malheureusement trop souvent délaissée, permettant de mettre en évidence des tendances significatives. Il s'agit aussi d'une comparaison internationale qui favorise un déplacement de regard très productif, par exemple lorsqu'on examine le cas français à l'aune des autres pays. Enfin, les auteurs entendent laisser leur place tout à la fois aux sujets et aux processus sociaux, aux comportements individuels et aux logiques sociétales : ils sont ainsi conduits à souligner les paradoxes générés au niveau sociétal par l'intérêt individuel de la poursuite d'études, paradoxe dit « écologique » pour signifier les traditionnels effets pervers ou d'agrégation, paradoxe lié notamment au fait que d'autres facteurs jouent au niveau macro-économique. Et cette description fine des similitudes et des différences permet de repérer des corrélations et d'articuler les niveaux macro et microsociologiques.

2. De ce fait, le livre, et c'est là sa seconde vertu, apporte de nombreux résultats de recherche, parfois inattendus. Les auteurs expriment eux-mêmes leur étonnement devant certains résultats, tels l'absence de corrélation forte entre inégalités des revenus et inégalités à l'école, la distance plus forte qu'on ne pouvait l'imaginer entre les sociétés et leur école ou encore la relation négative entre cohésion sociale et emprise du diplôme.

Ces résultats, formulés très nettement, parfois même quasiment comme des lois, sont autant de sources de réflexion : "plus les inégalités de performances sont faibles, plus l'influence de l'origine sociale des élèves sur leur performance est, elle aussi, faible » (p. 72), " les systèmes qui scolarisent beaucoup d'élèves longtemps sont plutôt favorables au niveau et à l'égalité des élèves» (p. 77), "moins le marché du travail est dynamique, plus l'emprise du diplôme est forte » (p. 135), « les sociétés se distinguent moins par leurs inégalités scolaires que par le rôle qu'elles attribuent à ces inégalités dans la distribution des individus dans la structure sociale » (p. 152), "là où l'emprise $d u$ diplôme est forte, non seulement les inégalités de carrières scolaires sont prononcées mais la reproduction, ici mesurée par l'immobilité des revenus, est plus prononcée » (p. 153), «la perception des inéga- lités, comme excessives, ne reflète pas des inégalités sociales objectives » (p. 166).

D'autres commentaires sont plus problématiques, surtout lorsqu'il s'agit de passer du constat à l'interprétation. Il en est ainsi de "l'impression de relative indépendance entre intégration, inégalités et cohésion scolaire » (p. 85) dont les causes possibles sont très diverses (le financement de l'éducation, l'organisation de l'école). De même, le fait que «les relations semblent moins systématiques entre les modèles d'organisation des systèmes éducatifs et la cohésion scolaire qu'elles ne le sont entre ces types d'organisation et les inégalités et les performances scolaires » peut révéler tout à la fois l'importance du «style éducatif», celle des conceptions du métier d'enseignant ou de la manière de travailler et de faire la classe. Et le constat selon lequel « la cohésion (...) se fonderait davantage sur des représentations que sur des faits » (p. 174) peut s'interpréter de diverses façons. Ainsi, en constatant que "plus l'emprise du diplôme est forte, plus la cohésion sociale est faible », les auteurs envisagent "une tension, sinon une contradiction, entre le rôle social et économique des diplômes, les avantages qui leur sont associés sur le marché du travail et la cohésion des sociétés » (p. 158).

Le livre est également riche de petites observations égrainées au fil du texte et qui incitent à prolonger la réflexion. Ainsi, en signalant que «les analyses de l'OCDE montrent qu'un dixième seulement de la variation des performances des élèves aux épreuves PISA tient spécifiquement au pays d'appartenance alors que les neuf dixièmes s'expliquent par leurs caractéristiques personnelles » (p. 71), ils mettent en doute les commentaires de cette enquête en termes de palmarès des pays. Et en constatant que « les pays diffèrent davantage par leurs modes d'articulation entre formations et emplois, entre les diplômes et le "rendement" qu'on en tire sur le marché du travail, que par l'ampleur des inégalités sociales devant la réussite scolaire » (p. 132), ils ouvrent la voie à d'autres perspectives interprétatives.

Enfin, l'ouvrage est parsemé de conseils sur l'éducation qui résonnent bien dans le contexte actuel : «il ne suffit donc pas de réduire les inégalités scolaires pour réduire les inégalités sociales » écrivent-ils 
page 155 ; " on peut donc militer pour une école plus compréhensive et plus accueillante sans penser pour autant que cette école là changera le visage de la société. A l'opposé, il n'y a pas à attendre que la société devienne meilleure pour améliorer l'école» (p. 179) ; «trop d'école tue l'école» (p. 190), « une meilleure école suppose sans doute qu'elle n'ait pas le monopole de la formation et de la répartition des individus dans la société (...) il ne faut donc pas renoncer aux valeurs les plus humanistes de l'éducation afin de former des individus épanouis et des citoyens solidaires, alors que l'emprise scolaire excessive, par sa force même et les paradoxes qu'elle engendre, compromet cet idéal » (p. 191). Le lecteur trouvera ainsi matière à entretenir ses convictions ou ses critiques.

3. Cet ouvrage soulève ainsi de nombreuses questions qui incitent à des prolongements de recherche. La première concerne la question des typologies. Si l'on veut éviter le relativisme, et certaines formules de l'ouvrage n'y échappent pas - « dans la famille des pays dynamiques et inégalitaires, l'école peut être à la fois intégratrice et inégalitaire » mais aussi peu intégratrice et égalitaire (p. 107) - il faut bien mettre un peu d'ordre dans cette variété extrême de configurations. Pour autant, les auteurs n'entendent pas proposer une typologie de plus mais préfèrent plutôt utiliser, confronter, valider les typologies courantes des systèmes nationaux, du type de celle d'EspingAndersen qui distingue pays libéraux, pays sociodémocrates et pays corporatistes, quitte à ajouter un autre type, en l'occurrence celui des pays méditerranéens ou ex-socialistes dits de capitalisme tardif. Il faut bien tenter de dépasser le constat de diversité qui risque de générer une fuite en avant dans la complexité et le sentiment inévitable que plus on approfondit plus on singularise. Les typologies ont donc leur intérêt, même si elles soulèvent de sérieux problèmes : sensibilité au choix des critères de distinction, impossible exhaustivité et besoin parfois de "forcer" les situations concrètes pour les faire entrer dans les cases, dimension statique qui ne permet pas de rendre compte du changement, etc..

On peut, à cette occasion, s'interroger sur leur rôle dans la recherche : sont-elles définies a priori ou $a$ posteriori dans une approche inductive ? Sont-elles des idéaux-types ou de simples cas ? Comment se construisent les critères de partition entre les types? Que faire finalement de ce foisonnement expansif de typologies? Et ne faudrait-il pas rechercher, selon un principe de parcimonie, une typologie transversale? "Une typologie pour être pertinente doit correspondre à plusieurs idéaux-types constitués en fonction de paramètres identiques dont les modalités seules varient » écrit D. Schnapper, dans La compréhension sociologique, démarche de l'analyse typologique (PUF, 1999). Une typologie, précise-t-elle, est donc une description mais aussi une interprétation et elle doit éviter deux écueils opposés : être trop abstraite et ne rien expliquer, être trop proche du concret et rester descriptive. Finalement, sa vertu première n'estelle pas d'ouvrir de nouvelles pistes de recherche et d'inciter à développer des travaux monographiques complémentaires ?

La seconde question est celle du comparatisme. Dans son article de Sociologie du Travail, - « Méthode comparative et analyse sociétale; les implications théoriques des comparaisons internationales ", $\mathrm{n}^{\circ} 2$, (1989) - M. Maurice distingue l'approche sociétale de deux approches courantes en matière de comparaison internationale. Dans les approches fonctionnalistes, « la comparabilité se fonde sur le principe de rationalité qui suppose une continuité entre les phénomènes que l'on compare, terme à terme, dans chaque pays »; la référence nationale n'est alors que contextuelle et il s'agit de trouver dans chaque pays des « équivalents fonctionnels " : il y a donc un postulat d'universalisme et de convergence des sociétés. Dans les approches culturalistes, "la référence "nationale" ne se réduit pas à un simple contexte mais elle est conceptualisée en termes de "culture nationale" ". Le risque alors est un faible pouvoir de généralisation et un biais de particularisme. Au contraire, l'analyse sociétale « compare de l'incomparable », recherche des « cohérences » nationales propres à chaque pays, privilégie l'analyse des processus d'interdépendance et des médiations, exclut toute comparaison terme à terme et évite ainsi les risques respectifs d'universalisme et de particularisme des deux approches précédentes.

Le risque du culturalisme affleure parfois de l'ouvrage lorsqu'il interprète les constats à, partir de l'histoire du pays, de sa culture, de ses "traditions éducatives nationales » : "la "fabrication" de la cohésion 
scolaire apparaît comme une alchimie procédant des traditions et des cultures de chaque pays » (p. 101); «ce sont moins les structures que les cultures qui sont emboîtées » (p. 107). Mais, dans d'autres passages, les auteurs semblent implicitement se référer à des explications plus « sociétales » : «la seule conclusion possible est la grande variété des situations et le fait que la cohérence des systèmes procède de constructions locales, engendrées par l'histoire économique, politique et sociale singulière de chaque pays" écrivent-ils page 114 .

Mais peut-on éviter les explications exogènes, parfois même $a d$ hoc ? Peut-on aller au-delà de supputations générales quand on ne dispose pour caractériser les pays que d'une série d'indicateurs ? Les auteurs sont conscients de la difficulté lorsqu'ils suggèrent, et pratiquent parfois sur quelques pays, une analyse plus approfondie des logiques nationales.

4. Enfin, la lecture de cet ouvrage incite à approfondir les interprétations des constats avancés car nombre de questions restent en suspens. Ainsi, qu'en est-il des processus et des évolutions ? Qu'en est-il des acteurs ? Peut-on passer d'un modèle à un autre et comment? Quelles explications peut-on avancer à toutes ces différences et similitudes ?

On peut ainsi, à la lecture, essayer de repérer des enchaînements de causes à travers l'articulation des typologies et le passage possible d'un type à un autre, ou encore apprécier le rôle respectif des variables interprétatives proposées. Ainsi, la faible influence des variables macro-économiques sur les attitudes individuelles est déjà une interprétation qui incite à rejeter des causalités globales fréquemment avancées. De même, le rôle décisif du marché du travail, de la place du diplôme, des relations entre formation et emploi est une clé interprétative possible qui ne peut que réjouir les lecteurs de cette revue. Mais il resterait à expliciter le rôle des acteurs, à intégrer dans l'analyse l'état des relations professionnelles ou le rôle du système éducatif, à propos duquel on voit pointer une opposition entre l'éducation, qui renvoie plutôt aux besoins de la société, et la formation, qui concerne plutôt les entreprises.

Bien d'autres constats restent à interpréter, comme l'influence du désajustement individuel entre formation et emploi sur la confiance et la tolérance. Et d'autres variables seraient à ajouter, comme le poids de la formation continue ou les spécificités de l'enseignement supérieur, si l'on veut mieux cerner cette notion d'emprise de l'école.

Autant de perspectives de recherche qui nécessiteraient d'entrer, au-delà de la comparaison des données statistiques, dans la spécificité de chaque pays en s'appuyant sur des monographies sélectionnées à partir des constructions typologiques. On pourrait alors mieux comprendre les processus sociaux et les jeux d'acteurs à l'origine de ces observations.

\section{Référence de l'ouvrage}

Dubet F., Duru-Bellat M., Vérétout A. (2010), Les sociétés et leur école: Emprise du diplome et cohésion sociale, Seuil. 